\title{
POSITIVE RESPONSE TO ANTI-HER THERAPY FOR METASTATIC MALIGNANT BREAST CANCER: CASE REPORT
}

Pedro Henrique de Ávila Perillo', Pedro Vinicyus Novais e Souza', Anelize Maria Bunholli', Fernando Santos de Azevedo², Lanúscia Morais de Santana², Lara Letícia Gaspar Sousa³, Julia Roberta Rodrigues de Faria ${ }^{4}$

${ }^{1}$ Faculdade de Medicina, Universidade Federal de Goiás - Goiânia (GO), Brazil.

${ }^{2}$ Centro Avançado de Diagnóstico de Câncer de Mama, Hospital das Clínicas, Universidade Federal de Goiás - Goiânia (GO), Brazil.

${ }^{3}$ Faculdade Unida de Campinas - Goiânia (GO), Brazil.

${ }^{4}$ Pontifícia Universidade Católica de Goiás - Goiânia (GO), Brazil.

Introduction: Progression-free survival in patients with HER2-positive breast cancer was significantly improved after administering dual block (DB) associated with docetaxel when compared with trastuzumab and docetaxel. This case report is about a patient with malignant breast cancer - HER2 positive - and metastatic lung and liver disease. Objective: The aim of this study is to report the positive response to the oncologic treatment. Case Report: A 43-year-old woman was diagnosed in 2011 with malignant breast cancer in early stage, i.e., cT1N0M0, with acute liver lesion not detected by tomography. She underwent upfront surgery, adjuvant chemotherapy with AC-TH schedule plus 1 year of Herceptin, and adjuvant radiotherapy. The tumor was developed again in 2015 through physical examinations, revealing metastatic lung and liver disease. She underwent first-line chemotherapy with docetaxel, Herceptin, and pertuzumab with DB maintenance. After additional physical examination, she presented an excellent response to treatment, remaining on maintenance with DB since October 2015 with an exceptional response to oncologic therapy. Discussion: It was observed an excellent response to treatment. Despite the poor prognosis, the patient presented a complete clinical improvement, and the treatment ensured much longer than average survival and an outstanding quality of life. Conclusion: Using docetaxel, Herceptin, and pertuzumab in patients with breast cancer, HER2 positivity improved the median overall survival of the patient; this confirms, albeit as an isolated case, the data from the Cleopatra study.

Keywords: Breast Cancer; Exceptional Response; Metastasis; Oncologic Treatment; anti-HER. 